\title{
The Solar Energetic Particle Event of 14 December 2006
}

\author{
T.T. von Rosenvinge · I.G. Richardson · D.V. Reames • \\ C.M.S. Cohen • A.C. Cummings • R.A. Leske • \\ R.A. Mewaldt • E.C. Stone • M.E. Wiedenbeck
}

Received: 2 January 2009 / Accepted: 31 March 2009 / Published online: 17 April 2009

(C) The Author(s) 2009. This article is published with open access at Springerlink.com

\begin{abstract}
The solar energetic particle event on 14 December 2006 was observed by several near-Earth spacecraft including the Advanced Composition Explorer (ACE), STEREO A and $\mathrm{B}, \mathrm{SOHO}$ and Wind. An interesting feature of this event is a series of unusual fluctu-
\end{abstract}

STEREO Science Results at Solar Minimum

Guest Editors: Eric R. Christian, Michael L. Kaiser, Therese A. Kucera, O.C. St. Cyr

T.T. von Rosenvinge $(\bowtie) \cdot$ I.G. Richardson · D.V. Reames

Code 661, NASA/Goddard Space Flight Center, Greenbelt, MD 20771, USA

e-mail: tycho.t.vonrosenvinge @ nasa.gov

I.G. Richardson

e-mail: ian.g.richardson@nasa.gov

D.V. Reames

e-mail: Donald.V.Reames@nasa.gov

I.G. Richardson

CRESST and Department of Astronomy, University of Maryland, College Park, MD 20742, USA

C.M.S. Cohen · A.C. Cummings · R.A. Leske · R.A. Mewaldt · E.C. Stone

290-17 Cahill Center, California Institute of Technology, Pasadena, CA 91125, USA

C.M.S. Cohen

e-mail: cohen@srl.caltech.edu

A.C. Cummings

e-mail: ace@srl.caltech.edu

R.A. Leske

e-mail: ral@srl.caltech.edu

R.A. Mewaldt

e-mail: rmewaldt@srl.caltech.edu

E.C. Stone

e-mail: ecs@srl.caltech.edu

M.E. Wiedenbeck

Jet Propulsion Laboratory, California Institute of Technology, Pasadena, CA 91109, USA

e-mail: mark.e.wiedenbeck@jpl.nasa.gov 
ations in the particle intensity that occurred during the first few hours. These fluctuations were observed inside a magnetic cloud that originated in a solar event on 13 December and show both similarities and variations at the different spacecraft. Interestingly, the most striking difference is between observations at the two closely-separated STEREO spacecraft. In particular, large fluctuations in the proton intensity were seen by the High Energy Telescope (HET) on STEREO A, and to a lesser extent at Wind and ACE, but not by the STEREO B HET. We conclude that the differences in intensity-time profiles were caused by anisotropies in the particle distribution and the different viewing directions of the individual particle telescopes. The intensity/anisotropy variations suggest that flux tubes with different particle propagation conditions existed within this magnetic cloud despite the absence of local magnetic field signatures associated with these regions. The intensity fluctuations are similar to those occasionally seen in impulsive particle events. There were also spacecraftto-spacecraft differences during the onset of the particle event. An initial rapid onset of energetic $(>40 \mathrm{MeV})$ protons was observed by the STEREO A and B spacecraft outside the magnetic cloud, but not by spacecraft such as SOHO that were already inside the magnetic cloud at this time. The latter spacecraft observed a slower, lower intensity increase. Evidently, energetic proton propagation from the solar event to the vicinity of Earth was inhibited within the magnetic cloud compared to outside.

Keywords Solar energetic particles $\cdot$ STEREO $\cdot$ Magnetic cloud

\section{Introduction}

The last group of major solar energetic particle (SEP) events of solar cycle 23 was observed in December 2006 as solar active region 10930 rotated across the visible hemisphere of the Sun. Particle events that included $>25 \mathrm{MeV}$ protons commenced on 5 December (associated with an X9.0 flare at E79 ${ }^{\circ}$ with X-ray peak emission at 10:35 UT), 6 December (X6.5, E63 ${ }^{\circ}, 18: 47$ UT), 13 December (X3.4, W23 ${ }^{\circ}, 02: 40$ UT) and 14 December (X1.5, W46 ${ }^{\circ}$, 22:15 UT). Halo CMEs were observed by the LASCO coronagraphs in association with the events of 13 and 14 December, with speeds of $1774 \mathrm{~km}$ and $1042 \mathrm{~km} \mathrm{~s}^{-1}$, respectively; the 5 and 6 December events occurred during SOHO/LASCO data gaps. The focus of this paper is the 14 December event. After launch on 26 October 2006, the STEREO A (Ahead) and B (Behind) spacecraft were, at the time of this event, close together upstream of the Earth's bow shock and approaching the Moon for a lunar swingby on 15 December (at 21:28 UT for STEREO A; 21:03 UT for STEREO B) that placed the spacecraft on separate trajectories, as shown in Figure 1.

\section{Observations}

Figure 2 shows an overview of energetic particle observations (13.6-100 MeV protons and 1.4-2.8 MeV electrons) from the HET instruments on STEREO A and B (von Rosenvinge et al., 2008) for the events of 13 and 14 December 2006. Typically, such SEP events originating on the western hemisphere of the Sun show a rapid increase in intensity followed by a slower decay (e.g., Cane, Reames, and von Rosenvinge, 1988). This was the case for the 13 December event which also showed an additional increase, more prominent in lower energy protons, associated with passage of the interplanetary shock from this solar event (14:38 UT on 14 December at STEREO). (See Liu et al., 2008 for a comprehensive 
Figure 1 Trajectories of STEREO A (red) and STEREO B (blue) after spacecraft launch and relative to the orbit of the Moon (orange) and the Earth's bow shock (green). The spacecraft location on 14 December is indicated. The ACE, SOHO and Wind spacecraft (not shown) were in orbit around the $L_{1}$ point $\sim 1.5$ million $\mathrm{km}$ upstream of the Earth.

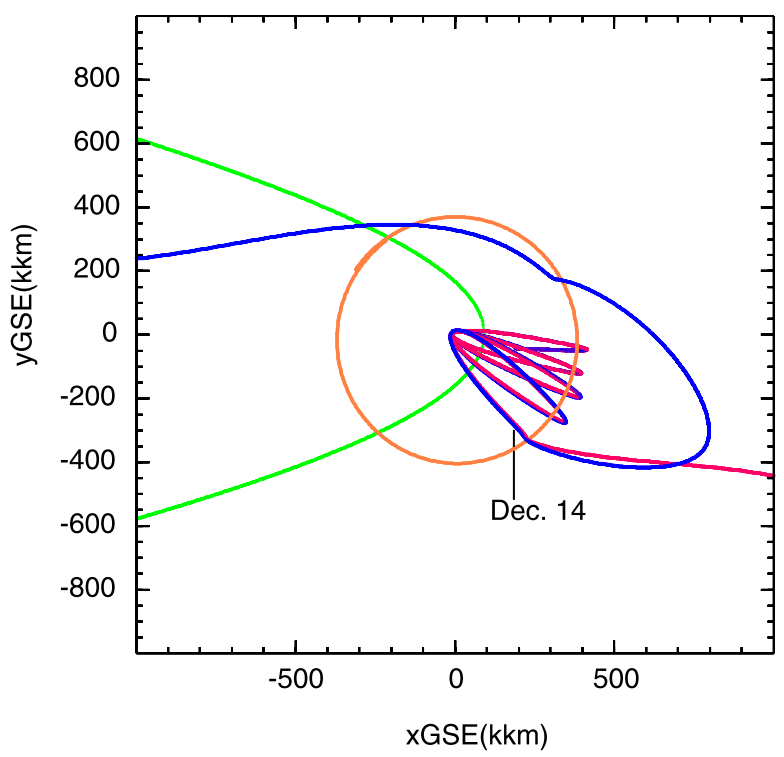

overview of the solar phenomena and interplanetary consequences of this event.) In contrast, the 14 December SEP event, which commenced during the decay of the previous event, exhibited unusual, abrupt fluctuations in intensity during the first few hours. These intensity fluctuations have been noted previously by Mulligan et al. (2008) and Mulligan, Blake, and Mewaldt (2008). However, the new feature of the observations that we discuss here, evident in Figure 2, is that the fluctuations were larger, and extended over a longer interval, in HET observations at STEREO A than at STEREO B even though the two spacecraft were close together at this time, separated by only $\sim 7200 \mathrm{~km}$. The fluctuations were also more pronounced in protons than in electrons. Note also that maximum proton intensities early in the 14 December event were nearly two orders of magnitude lower than in the 13 December event even though the 14 December solar event was nominally better magnetically connected to the Earth. The X-ray flare intensities were also similar. However, the associated CME was considerably slower on 14 December $\left(1042 \mathrm{~km} \mathrm{~s}^{-1}\right)$ than on 13 December $\left(1774 \mathrm{~km} \mathrm{~s}^{-1}\right)$. In this paper, we investigate the origin of these intensity fluctuations, and the reason for the different intensity profiles at the two STEREO spacecraft.

Figure 3 compares the intensity fluctuations for $13.6-15.1 \mathrm{MeV}$ and $60-100 \mathrm{MeV}$ protons at STEREO A (HET A) and B (HET B). The initial particle increase following the 14 December solar event was seen earlier in the higher energy range, as expected for velocity dispersion; there was also a higher background at the lower energy associated with the 13 December event and the related shock. Initially, both HETs saw similar intensity fluctuations, but after $\sim 03$ UT on 15 December, the fluctuations in both energy ranges were substantially larger at HET A. In particular, the HET A intensity at $60-100 \mathrm{MeV}$ decreased to around a third of the intensity in HET B while the change at $13.6-15.1 \mathrm{MeV}$ was around an order of magnitude (i.e., the HET A proton spectrum was harder in the intensity decreases). At both energies, when the HET A intensity recovered temporarily during the fluctuations, it closely matched the intensity in HET B. The fluctuations tended to decrease in amplitude with time, and ceased at $\sim 08$ UT. They were also essentially dispersionless, suggesting that they were spatial, rather than temporal, in origin. 

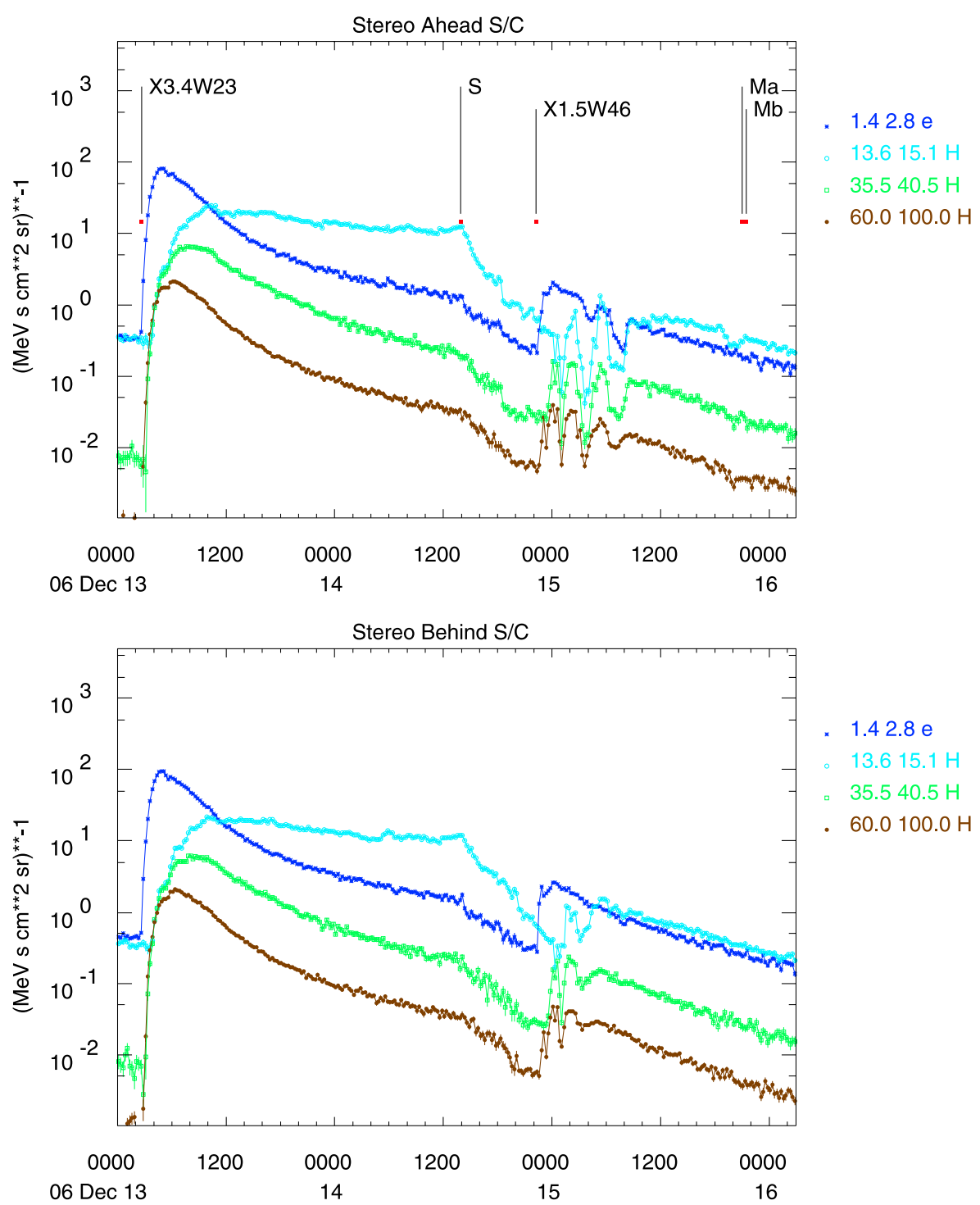

Figure 2 Observations of 13.6-100 MeV protons and 1.4-2.8 MeV electrons from the HET instruments on STEREO A (ahead) and B (behind) for the events of 13 and 14 December 2006. The times of shock passage (S) on 14 December and the closest approaches of STEREO A and B to the Moon (Ma and Mb) are also indicated.

We first consider whether these fluctuations were local to the STEREO spacecraft and in particular whether the proximity of the spacecraft to the Moon was involved in some way. Figure 4 shows ion intensities at various energies from both STEREO HETs and from the EPAM and SIS instruments on the Advanced Composition Explorer (ACE) spacecraft and the EPACT instrument on Wind. Both ACE and Wind were in orbit around the $L_{1}$ Lagrangian point $\sim 1.5$ million $\mathrm{km}$ upstream of the Earth, and far ( $\sim 3$ Earth - Moon distances) upstream of the orbit of the Moon. Similar intensity modulations were observed at Wind and ACE, 


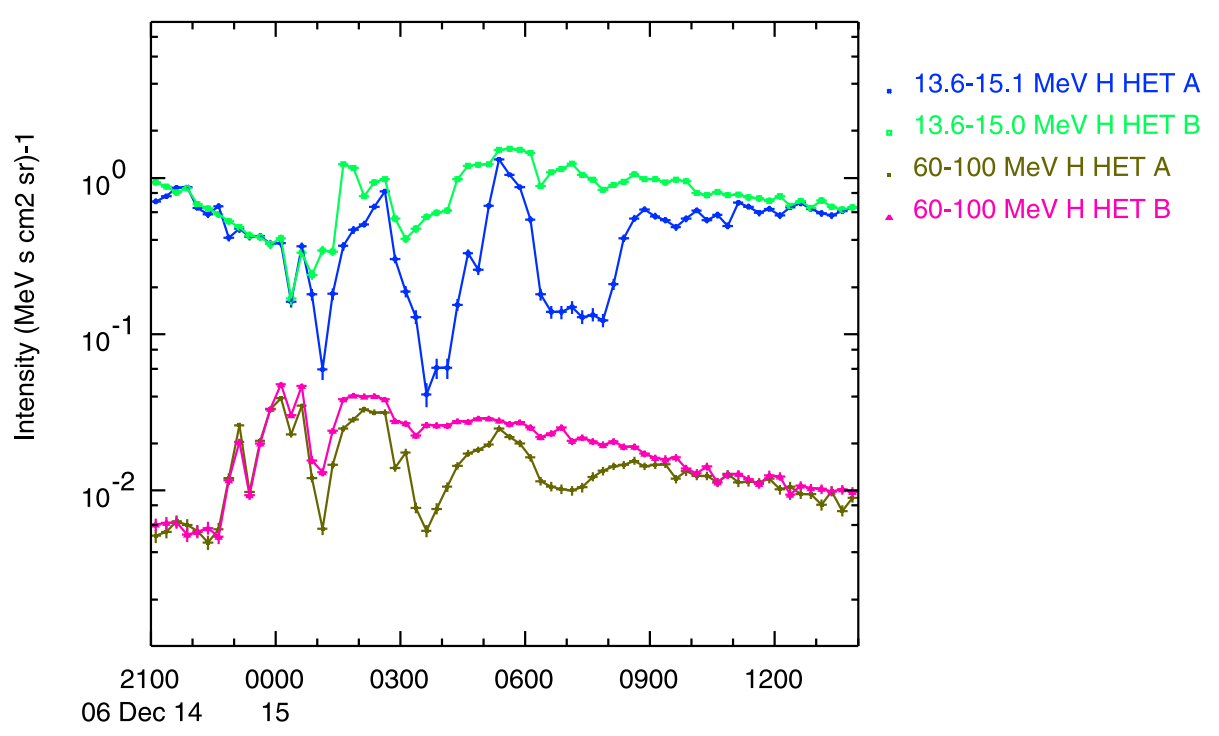

Figure 3 15-minute averaged intensities of 13.6-15.1 MeV and 60-100 MeV protons observed by HET A and B from 21 UT on 14 December to 14 UT on 15 December.

albeit of lower amplitude, as at STEREO A, indicating that these intensity variations were not local to STEREO but were observed over a large region in the vicinity of Earth (see also Mulligan, Blake, and Mewaldt, 2008). In addition, no comparable fluctuations were observed as the STEREO spacecraft approached the Moon (Figure 2), also suggesting that the Moon did not play a role in their production. Furthermore, close inspection of Figure 4 suggests that the intensity modulations were observed first at ACE or Wind then, with a delay of $\sim 20-30$ minutes, at STEREO.

Since the STEREO spacecraft are 3-axis stabilized, the HETs view in fixed directions. Hence, one possibility for producing intensity fluctuations at the two spacecraft is that the HETs were viewing an anisotropic beam of particles traveling along the magnetic field, and changes in the field direction brought particles of different pitch angles into the telescope viewing cones (full width $=55^{\circ}$ ), varying the observed intensity. The bottom panels of Figure 4 show however that the field direction was only changing slowly during the period in which the intensity fluctuations were present. In particular, the intensity variations were generally not correlated with the field variations. Hence, changes in magnetic field direction relative to the viewing directions of the HET telescopes alone were not responsible for the intensity variations seen at STEREO.

As has also been noted by Mulligan et al. (2008) and Mulligan, Blake, and Mewaldt (2008), the particle intensity variations and smooth magnetic fields observed by near-Earth spacecraft occurred during passage of the interplanetary coronal mass ejection (ICME) driving the shock on 14 December that was related to the 13 December solar event. Figure 5 illustrates solar wind observations from ACE that show evidence of the presence of the ICME (bounded by the purple vertical lines), which had the enhanced magnetic field and smooth rotation in direction characteristic of a "magnetic cloud" (Klein and Burlaga, 1982; Mulligan et al., 2008; Liu et al., 2008), the upstream shock (green vertical line) and the intervening sheath region. Additional signatures typical of an ICME/magnetic cloud (e.g., Zurbuchen and Richardson, 2006 and references therein) that were present include: $\mathrm{Ab}$ - 


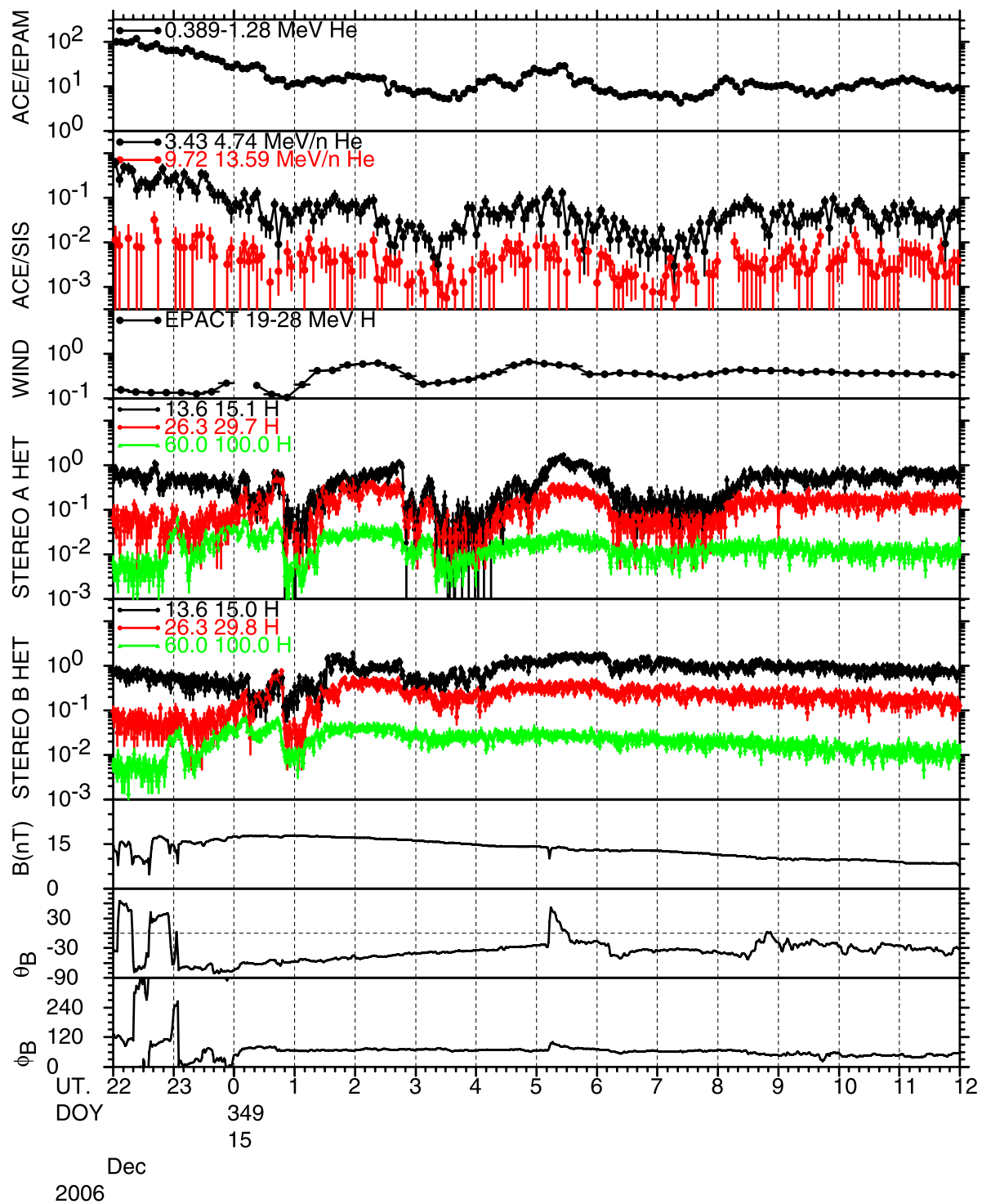

Figure 4 Energetic particle observations from Wind, ACE and STEREO illustrating that the intensity variations at STEREO were also observed around 20 minutes earlier at Wind and ACE, in orbit around $L_{1}$ (intensities are in units of $\left.\left(\mathrm{MeV} / \mathrm{n} \mathrm{s} \mathrm{cm}^{2} \mathrm{sr}\right)^{-1}\right)$. The bottom panels show the magnetic field intensity and polar and azimuthal angles at STEREO A in GSE coordinates.

normally low solar wind proton temperatures $\left(T_{\mathrm{p}}\right)$ compared to expected values for normal solar wind $\left(T_{\mathrm{ex}}\right)(\mathrm{red})$ where black shading indicates when $T_{\mathrm{p}}<0.5 T_{\mathrm{ex}}$ a common indicator of an ICME (see Richardson and Cane, 1995 for details); a descending speed profile; high solar wind He/p ratio (though this was not confined to the magnetic cloud as defined here); enhanced solar wind ion charge states (the $\mathrm{O}^{7} / \mathrm{O}^{6}$ ratio and mean Fe charge state are shown here) measured by the SWICS instrument on ACE; solar wind compositional anom- 


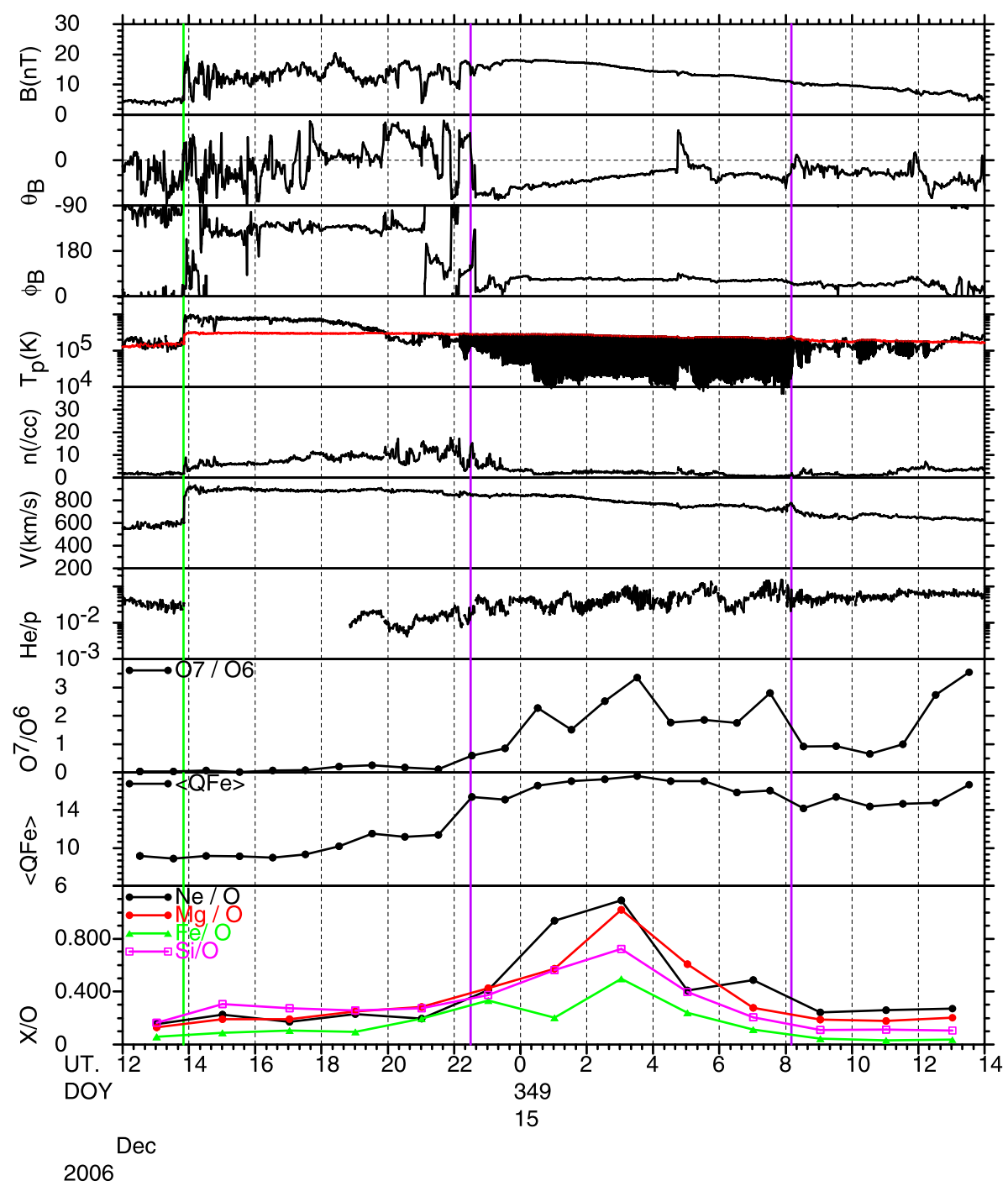

Figure 5 ACE solar wind magnetic field and plasma observations indicating passage of the shock (green vertical line) and ICME (bounded by the vertical purple lines) associated with the 13 December solar event.

alies, also observed by SWICS; and bidirectional suprathermal electrons (see the top panel of Figure 6 and also Liu et al., 2008). Note that the 14 December solar event (at 22:15 UT) occurred just as the leading edge of the fast $\left(\sim 800 \mathrm{~km} \mathrm{~s}^{-1}\right)$ magnetic cloud arrived at the vicinity of Earth $(\sim 22: 38 \mathrm{UT}$ at ACE, based on the time of the last field direction change prior to the smooth field region). The consequences of this near coincidence on the initial development of the related solar particle event will be discussed in Section 4.

Liu et al. (2008) estimate that the cloud axis direction was $\theta=-57^{\circ}, \phi=81^{\circ}$ in GSE coordinates, while the Wind magnetic cloud list (http://lepmfi.gsfc.nasa.gov/mfi/mag_cloud_ S1.html) gives an axis orientation of $\theta=27^{\circ}, \phi=85^{\circ}$. Thus, both agree that the axis was closely west to east but differ in whether it was inclined north or south, most likely because 

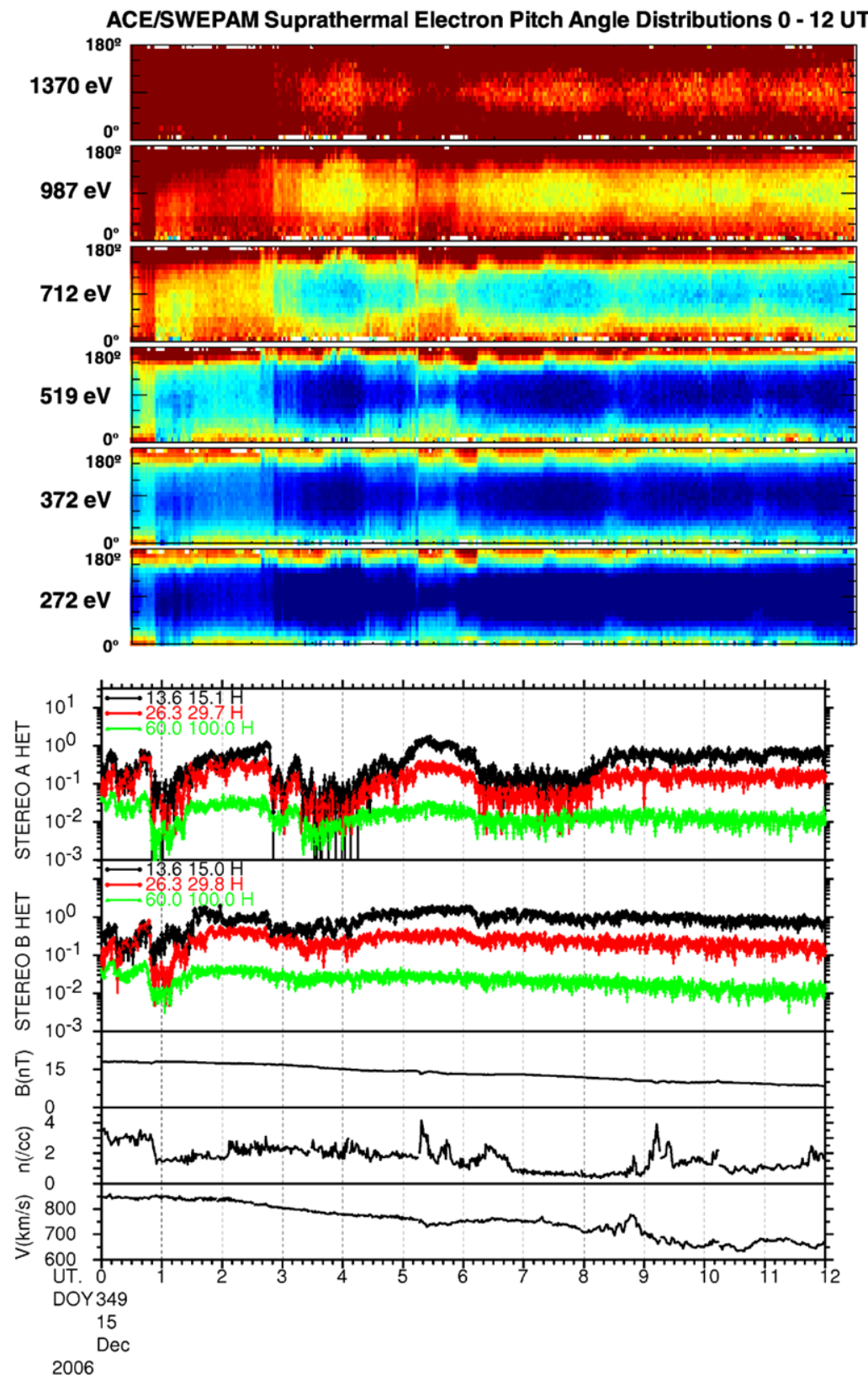

Figure 6 The top panel shows suprathermal electron $(272-1370 \mathrm{eV})$ pitch-angle distributions from the SWEPAM instrument on ACE for 00-12 UT on 15 December that indicate the presence of bi-directional field-aligned suprathermal electron flows. (Dark red indicates the highest intensities, dark blue the lowest.) Lower plots show energetic proton intensities from the STEREO A and B HETs, and the near-Earth magnetic field intensity and solar wind density and speed (from the OMNI database). The ACE electron pitch-angle plots have been offset by $\sim 30$ minutes to allow for the transit time from $L_{1}$ to the vicinity of the Earth. Note that the electron pitch-angle distribution tends to broaden when both STEREO HETs observe similar energetic proton intensities. 
different intervals were considered in their analyses. (Both started at 22:48 UT on 14 December, but ended at 04:34 UT and 19:48 UT on 15 December, respectively.) Both agree that the field was left-handed. Comparing Figures 4 and 5 it is evident that the SEP intensity variations under investigation were confined to the interior of the magnetic cloud and ceased close to the trailing edge.

Figure 6 compares the suprathermal electron pitch-angle distributions from ACE/ SWEPAM (http://www.srl.caltech.edu/ACE/ASC/DATA/level3/swepam/) with HET proton observations at STEREO A and B for 00-12 UT on 15 December 2006. The time scales are offset by $\sim 30$ minutes to allow for the transit delay between $L_{1}$ and STEREO at the observed solar wind speed of $\sim 800 \mathrm{~km} \mathrm{~s}^{-1}$ ( $c f$. the bottom plot of Figure 6). The suprathermal electron pitch angles from ACE show predominantly bi-directional, field-aligned streaming (which extended beyond our estimate of the magnetic cloud trailing edge) with features that appear to be related to the energetic proton intensity features at STEREO. In particular, the electron pitch-angle distributions tend to narrow at times when the intensity in HET A decreased relative to the intensity in HET B. There are also sharp changes in the electron distributions, e.g., at $\sim 01 \mathrm{UT}$ and $03 \mathrm{UT}$, that are associated with abrupt drops in the energetic proton intensity. This suggests that the proton intensity variations may reflect changes in general particle propagation conditions in the solar wind. The abrupt changes in the electron distribution and proton intensity at $\sim 01$ UT were associated with a decrease in the solar wind density, also shown in Figure 6 . There were several additional variations in the solar wind density during the period in Figure 6, but these show no clear association with the particle intensity variations.

\section{Why Do the STEREO A and B HETs Show Different Intensity-Time Profiles?}

A key to understanding the different HET intensity-time profiles at the two STEREO spacecraft is that, at this phase of the mission, STEREO B was inverted relative to its normal operating configuration, to allow the spacecraft antenna to point towards the Earth. (In its final orbit, STEREO B lags behind the Earth and the antenna points to the west. However, the STEREO spacecraft were ahead of the Earth on 14-15 December 2006 (Figure 1).) The HET telescopes are designed to point in a fixed direction along the nominal Parker spiral field $\left(\sim 45^{\circ}\right.$ west of the spacecraft-Sun line at $\left.1 \mathrm{AU}\right)$ when in their final orbit. Thus, on 14-15 December, the STEREO A HET was pointed, as intended, along the nominal spiral direction. However, the STEREO B HET was pointed in a direction $\sim 45^{\circ}$ east of the spacecraft-Sun line. Hence, the HETs were making particle observations in approximately orthogonal directions, raising the possibility that the different intensities measured by the two telescopes reflect anisotropies in the particle distribution. Figure 7 compares the HET A and B viewing directions and the magnetic field azimuth at STEREO A (in GSE coordinates; the field direction at STEREO B is essentially identical and not shown) from 18 UT on 14 December until 12 UT on 15 December. The directions of the nominal inward and outward Parker spirals are also indicated. Assuming a nominal Parker spiral field, we would expect SEP particles to have arrived in the viewing cone of HET A. However, the magnetic field during much of this interval was directed far from the Parker spiral direction. In particular, the field within the magnetic cloud (bounded by the vertical purple lines) was directed approximately $70^{\circ}$ east of the spacecraft - Sun line and lay close to the edge of the HET B viewing cone $\left(55^{\circ}\right.$ full width) indicated by gray shading in Figure 7 . On the other hand, contrary to the expectations for a Parker spiral field, HET A was viewing approximately perpendicular to the local magnetic field azimuth. 


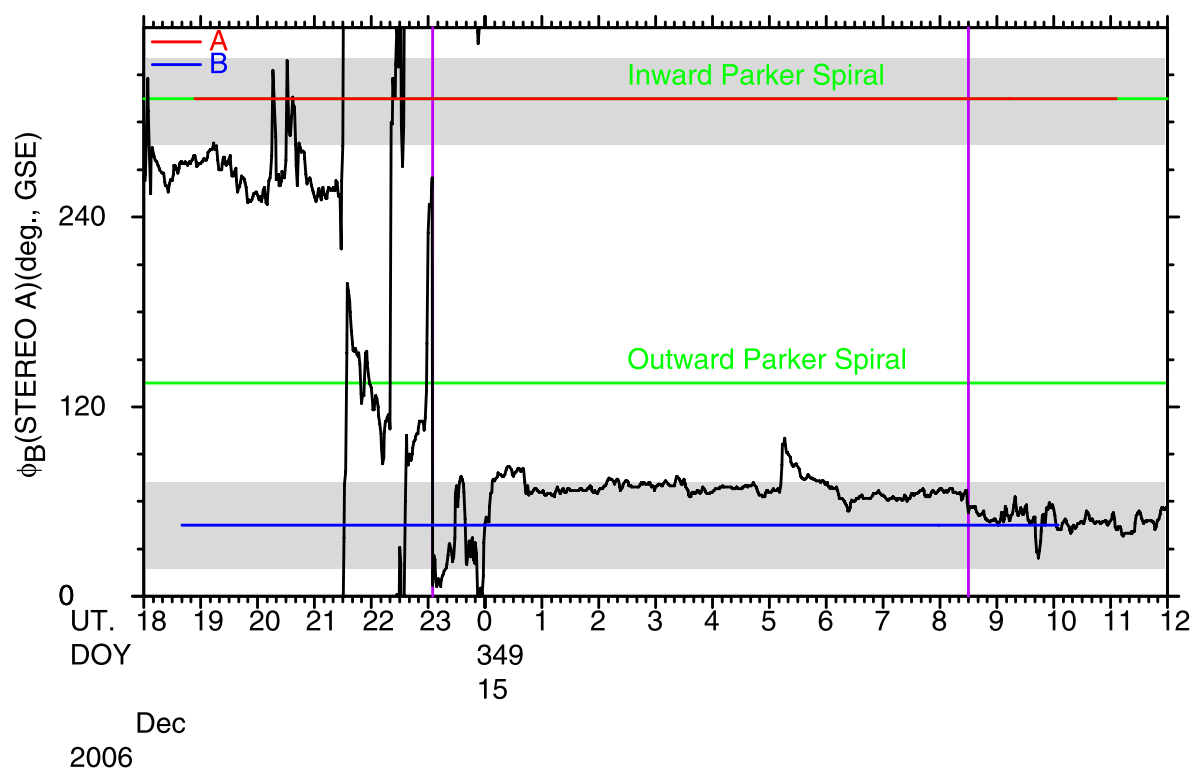

Figure 7 STEREO A and B HET viewing directions compared with the magnetic field azimuthal angle (at STEREO A in GSE coordinates) for 18 UT on 14 December to 12 UT on 15 December. Gray shading indicates the extent of the HET viewing cones. The nominal inward and outward Parker spiral directions are also indicated. Vertical purple lines mark the magnetic cloud boundaries.

The polar angle of the magnetic field should also be taken into consideration since the field was directed strongly southward at the beginning of the magnetic cloud and then returned towards the ecliptic (e.g., Figure 4). Figure 8 compares the angle (pitch angle) between the magnetic field direction and the HET A and B viewing directions from 23 UT on 14 December to 12 UT on 15 December. Because of the large southward field inclination, both HETs were sampling large pitch-angle $\left(\sim 70^{\circ}\right)$ particles inside the leading edge of the magnetic cloud. Subsequently, the field direction moved to within $\sim 30^{\circ}$ of the HET B viewing direction, close to the edge of the HET B viewing cone (black horizontal line). In contrast, the field was $\sim 100^{\circ}$ from the viewing direction of HET A during much of the period in Figure 8.

The next question is which ends of the magnetic field lines passing the spacecraft were connected to the Sun and guided particles to the spacecraft. This question is complicated by the field orientation being far from the Parker spiral and by the presence of counterstreaming suprathermal electrons which suggests that looped field lines were present with both ends rooted at the Sun. The suprathermal electron pitch-angle distributions in Figure 6 provide a clue in that the electron beam anti-parallel to the field direction (pitch angles $\sim 180^{\circ}$ ) appears to have been slightly stronger than that parallel to the field. This suggests that the suprathermal electron flow was preferentially from a direction east of the spacecraft-Sun line for much of the period in the magnetic cloud. If this were also the case for the energetic particles associated with the 14 December solar event, then within the magnetic cloud these particles would have been propagating anti-parallel to the magnetic field and into the field of view of HET B, while HET A would have observed particles with larger pitchangles $\sim 90^{\circ}$. If the particles were anisotropic and streaming along the magnetic field, we would then expect HET B generally to observe higher intensities than HET A. Furthermore, 


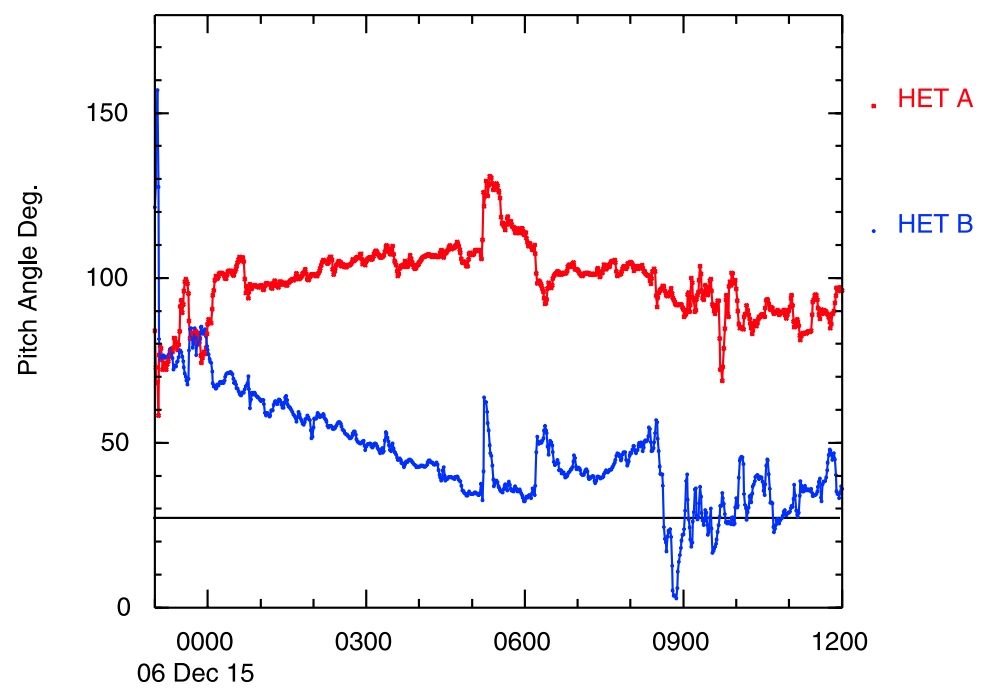

Figure 8 Angle between the magnetic field direction and STEREO A and B HET viewing directions (pitch angle), indicating that HET B was more closely aligned with the field direction during much of the period of interest than HET A, which was observing particles with pitch angles $\sim 90^{\circ}$. The black line indicates the edge of the HET viewing cone.

if the width of the particle pitch-angle distribution changed with time, we might expect the intensity at large pitch angles to show a stronger response, consistent with the larger variations in the proton intensity seen at HET A within the magnetic cloud. Since similar proton intensities were seen in both HETs when the intensity recovered between each modulation (Figure 3), the observations suggest a situation where the proton distribution was approximately isotropic in the higher intensity intervals, but was more beamed along (anti-parallel to) the magnetic field in those intervals where the HET A intensity was depressed.

The $2.5-4 \mathrm{MeV}$ proton intensity and anisotropy observations in Figure 9 from the LEMT instrument on Wind support the scenario in which particles were arriving close to the viewing cone of HET B and were more anisotropic during the intensity depressions. The LEMT anisotropy observations were obtained by binning particle counts into 16 azimuthal sectors. In the data discussed here, the start of sector $1\left(0^{\circ}\right)$ is defined by the azimuth of the magnetic field measured at the spacecraft with the sector number/azimuthal angle increasing in an anti-clockwise direction as viewed from north of the ecliptic. The top panel of Figure 9 summarizes the azimuthal variations in particle count rate in the spacecraft frame from 15 December, 00 UT to 10 UT in 15 minute intervals after fitting the sectored count rates by a 3rd-order Fourier series and normalizing to the maximum count rate. (Note that the angle relative to the magnetic field direction is defined differently in Figures 6 and 9. In particular, $0^{\circ}$ corresponds to particle flow anti-parallel to the field in Figure 9 but parallel to the field in Figure 6.)

As discussed above in relation to Figure 4, the particle intensity fluctuations were also observed at Wind. The spin-averaged counting rate in the third panel of Figure 9 illustrates that these fluctuations were also evident in 2.5-4 MeV protons. (Liu et al., 2008 show that they were also observed down to the lowest energy proton channel of the EPAM instrument on ACE (47-66 keV) i.e., 3 orders of magnitude in energy below the HET data discussed in this paper.) The angular distributions indicate that maximum particle counts (white/pink 


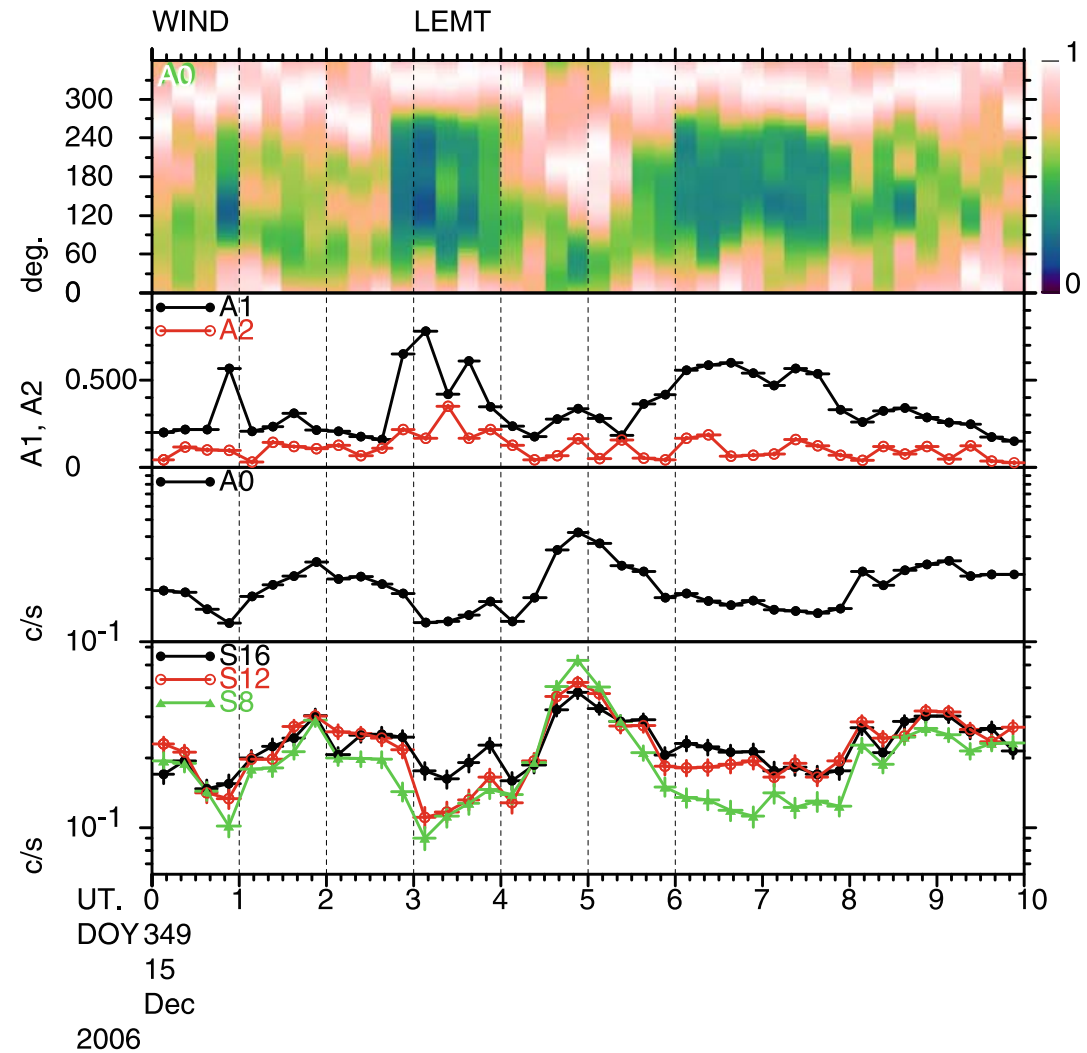

Figure 9 Observations of 2.5-4 MeV protons (15 minute averages) from LEMT on Wind. The top panel shows the time variation of the angular particle distribution observed in 16 azimuthal sectors. The viewing direction of each sector relative to the magnetic field direction is plotted on the left vertical axis, where $0^{\circ}$ indicates particles arriving anti-parallel to the field direction. The number of counts in each sector, relative to the count in the sector with the most counts, is displayed as a color (see the color axis to the right of the top panel). The second panel shows the amplitudes of the first and second Fourier harmonics of the particle angular distribution. The third panel shows the spin averaged counting rate in counts/second (A0). The bottom panel shows the counting rates in sectors detecting particles arriving anti-parallel to the magnetic field (S16, similar to the configuration of HET B), particles with pitch angles at $\sim 90^{\circ}$ (S12, similar to the configuration of HET A) and in sector 8, viewing in the opposite direction to S16. The protons predominantly arrived anti-parallel to the field (i.e., from east of the Sun-Earth line), and the degree of anisotropy increased during the proton intensity decreases.

shading) were generally observed at $\sim 300-330^{\circ}$ in the spacecraft frame. This is consistent with particle flow anti-parallel to the field from a direction east of the spacecraft-Sun line together with a radial Compton-Getting anisotropy (Forman, 1970), as discussed further below. For a brief period around $\sim 5$ UT the flow reversed direction. Since there was no field azimuth reversal at this time ( $c f$., Figure 7 ), this was most likely a true flow reversal. The angular distribution also became narrower at times when the counting rate decreased. These changes in the distribution are summarized in the second panel by the amplitudes of the first and second-order Fourier components. The first order (streaming) component was $\sim 20 \%$ in the higher intensity intervals. Subtraction of the Compton-Getting anisotropy $(\sim 10 \%)$ leaves a small anisotropy of $\sim 10 \%$. Thus, the $\sim 3 \mathrm{MeV}$ proton distribution was nearly isotropic in the solar wind frame in the high intensity intervals. In the low intensity intervals, 
the distribution was more anisotropic with a first order component of $\sim 60 \%$ or $\sim 50 \%$ with the Compton-Getting component removed. Thus, the Wind anisotropy variations are consistent with our conclusions above based on HET observations, albeit at energies that differ by a factor of $\sim 5$.

The bottom panel of Figure 9 illustrates how the LEMT counting rates varied in a sector viewing approximately along the field (sector 16) (i.e., detecting particles with small pitch angles arriving anti-parallel to the field, similar to the configuration of HET B), and sectors viewing perpendicular to the field (S12, similar to the viewing direction of HET A) and viewing anti-parallel to the field (S8). It is clear that the counting rate variations with time were larger in S12 and S8 than in S16, resembling the differences in the intensity variations measured by HET A and B though at a lower energy.

Bi-directional field-aligned energetic particle flows are often found in magnetic clouds and other ICMEs (e.g., Palmer, Allum, and Singer, 1978; Marsden et al., 1987; Richardson and Reames, 1993). Although the HET observations in only two viewing directions do not rule out the possibility that the detected protons may have developed bi-directional flows, the Wind anisotropy data suggest that only uni-directional or weak streaming occurred, at least at proton energies of $\sim 3 \mathrm{MeV}$.

Proton arrival along field lines directed from east of the spacecraft-Sun line and $\sim$ perpendicular to the nominal Parker spiral, as observed here, frequently occurs inside ICMEs/magnetic clouds but rarely outside these structures. In particular, Richardson and Cane (1996) found that particles in $\sim 30 \%$ of solar particle event onsets observed inside ICMEs/magnetic clouds at $0.3-1 \mathrm{AU}$ arrived from east of the spacecraft - Sun line because of the non-spiral fields in these structures and the possibility that field lines may be looped with both ends rooted at the Sun, allowing for particles to arrive via either (or both) of the legs of the field lines. In the case discussed here, considering the configuration of the magnetic cloud and the arrival direction of the particles, we suggest that the particles propagated to the vicinity of the Earth along the eastern "leg" of the magnetic cloud.

The reduced amplitude of the intensity variations at Wind and ACE compared to HET A in Figure 4 can also be accounted for if anisotropies were involved. Wind and ACE are spinning spacecraft, and the intensities plotted in Figure 4 are direction-averaged. Figure 9 illustrates that the relative intensity variations in sectors observing large pitch angle particles (similar to HET A) can be larger than in the spin average.

Inspection of Figures 4 and 6 suggests that the HET intensity shows finer scale features superposed on the prominent intensity variations on time scales of $\sim 2$ hours. Figure 10 compares 60-s averages of the HET A and B 13.6-15.1 MeV proton intensities during a one hour period (02:40 - 03:40 UT) on 15 December. The HET intensities show variations on time scales of the order of 5-10 minutes that are suggestive of changes in anisotropy from near-isotropic, such as from $\sim$ 03:08 to 03:18 UT when both telescopes saw similar intensities, to anisotropic during adjacent periods when the intensities differed by up to an order of magnitude. These features suggest the existence of structures with different ion propagation characteristics within the magnetic cloud with sizes of the order of 10 minutes $\times V_{\mathrm{sw}}\left(\sim 800 \mathrm{~km} \mathrm{~s}^{-1}\right) \sim$ half a million $\mathrm{km}(\sim 0.003 \mathrm{AU})$, compared with $\sim 6$ million $\mathrm{km}$ ( $0.04 \mathrm{AU})$ for the larger scale $(\sim 2$ hour) variations. However, there is no evidence in the STEREO magnetic field of local features with similar time scales ( $c f$. Figure 4; we cannot examine the presence of plasma features at STEREO because the plasma instruments were not yet in operation at this time.) This raises the possibility that the intensity variations were produced by propagation conditions between the Sun and 1 AU and remote from the observing spacecraft. 


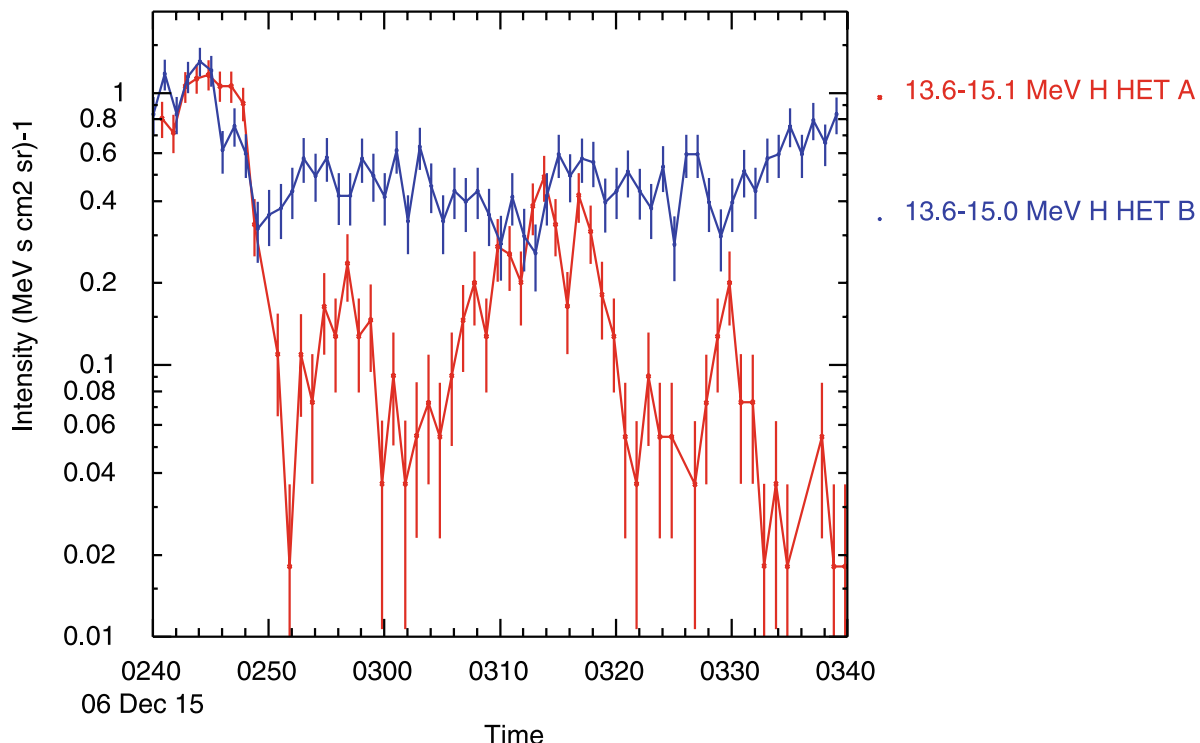

Figure 10 60-s averages of the HET A and B 13.6-15.1 MeV proton intensity during a one-hour period (15 December, 0240 - 0340 UT) showing evidence of structures on time scales of $\sim 5-10$ minutes.

\section{Observations During the Particle Event Onset}

Early in the particle event ( $<03$ UT, 15 December), both spacecraft saw comparable intensity modulations which then tapered off at STEREO-B (Figure 3). Figure 11 shows HET A and $\mathrm{B}$ observations of $0.7-1.4 \mathrm{MeV}$ electrons and $40-60 \mathrm{MeV}$ and $60-100 \mathrm{MeV}$ protons (60-s averages) prior to 03 UT together with the magnetic field azimuth (again at STEREO A in GSE coordinates; STEREO B is essentially identical and not shown) with the viewing directions of the HETs indicated. There are several interesting features during the onset of the particle event. Considering the energetic electron onset (second panel), the magnetic field at this time was initially aligned with the viewing direction of HET A. Consistent with this, and assuming field-aligned electron streaming, the intensity was slightly higher in HET A (red curve) than HET B (blue curve) for a very brief period. The field direction then moved to the east, and became closer to the viewing direction of HET B, resulting in a higher intensity in HET B than in HET A for a period of $\sim 20$ minutes. The field then returned briefly towards the HET A viewing direction before settling in a direction close to the HET B viewing direction (though with a large southward component, $c f$. Figure 4). We suggest that the last major change in field direction, indicated by the purple vertical line, was probably associated with passage of the leading edge of the magnetic cloud at STEREO. The subsequent, essentially identical electron intensities in both HETs suggest that the electron distribution may have been $\sim$ isotropic inside the magnetic cloud.

The STEREO energetic proton data show an initial rapid increase in intensity following the solar event, more prominent at $60-100 \mathrm{MeV}$, but this was terminated abruptly at the passage of the probable magnetic cloud leading edge. We conclude that particles from the 14 December solar event were unable to arrive promptly inside the magnetic cloud. A slower increase then occurred within the magnetic cloud. The similar intensities in HET A and B suggest that the particle distribution may have been nearly isotropic even in the initial increase, at least until the strong modulations in intensity occurred. As discussed above, these 


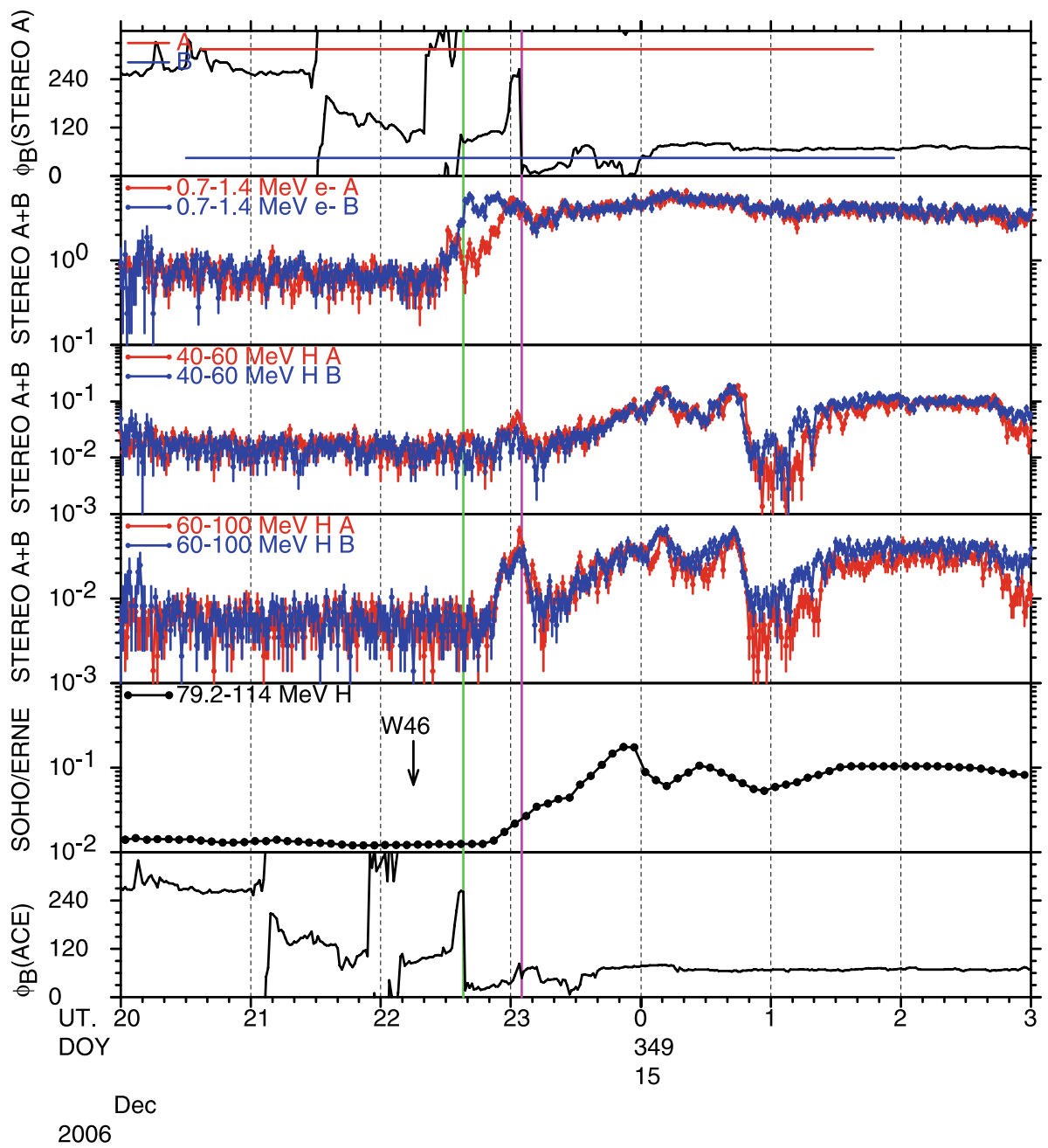

Figure 11 Summary of energetic particle observations and the magnetic field azimuth at the STEREO spacecraft and $L_{1}$ (SOHO/ACE) during the onset of the 14 December SEP event. The top panel shows the magnetic field azimuthal angle (GSE) at STEREO A (STEREO B is essentially identical and not shown) together with the HET viewing directions. Below are the STEREO A and B HET 0.7-1.4 MeV electron and 40-60 MeV and $60-100 \mathrm{MeV}$ proton intensities $\left(\mathrm{cm}^{2} \mathrm{sr} \mathrm{MeV} \mathrm{s}\right)^{-1} ; 60$-s averages). The bottom two panels show the $79.2-114 \mathrm{MeV}$ proton intensity measured by the ERNE instrument on SOHO and the magnetic field azimuth (GSE) at ACE, both at $L_{1}$. The green (purple) vertical lines indicate the probable arrival times of the leading edge of the magnetic cloud (last major field direction change) at $L_{1}$ and then at the STEREO spacecraft. A rapid high energy proton increase was observed at the STEREO spacecraft outside the magnetic cloud, but not at SOHO inside the magnetic cloud. This increase was terminated as the STEREO spacecraft entered the magnetic cloud. A slower increase, similar to that at SOHO was then observed. Variations in the HET A and B electron intensity during event onset were associated with changes in the magnetic field direction, suggesting that the electron distribution was anisotropic. After arrival of the magnetic cloud, the similar intensities in both telescopes suggest that the electron distribution was $\sim$ isotropic at least until the end of the interval shown here. 


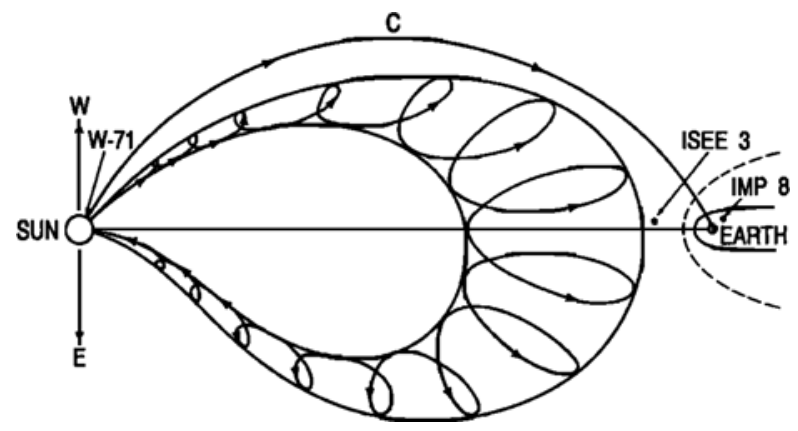

Figure 12 Configuration of the IMP 8 and ISEE 3 spacecraft on 16 February 1980 ahead of an approaching magnetic cloud. As discussed by Farrugia et al. (1993), observations of a particle onset that occurred shortly before the magnetic cloud moved over the spacecraft demonstrated that the particles arrived more slowly inside the magnetic cloud than outside, possibly delayed by the longer path length along the helical field lines in the magnetic cloud. A similar situation may have occurred in the 14 December 2006 event except that in this event, the particles were probably guided to the vicinity of the Earth along the eastern leg of the magnetic cloud.

modulations were larger at HET A than at HET B consistent with an anisotropic distribution arriving along a magnetic field that was approximately aligned with the HET B viewing direction.

It is interesting to compare the STEREO observations during event onset with similar observations from spacecraft in orbit around the upstream $L_{1}$ point. We would expect the magnetic cloud to have arrived earlier at $L_{1}$ than at STEREO, and this was the case. The bottom panel of Figure 11 shows the magnetic field azimuth at ACE. This includes several variations in direction that are similar to those at STEREO but displaced in time. If we again take the final major direction change as the leading edge of the magnetic cloud (green vertical line), this passed ACE $\sim 25$ minutes earlier than at STEREO. As a consequence, spacecraft at $L_{1}$ would have been inside the magnetic cloud at the time when STEREO (outside the magnetic cloud) observed the initial rapid increase in high energy proton intensity. Since at STEREO this increase was abruptly terminated by arrival of the magnetic cloud, suggesting that these particles were excluded from the magnetic cloud, we might expect that spacecraft at $L_{1}$ would not have detected the rapid increase seen at STEREO. The $79.2-114 \mathrm{MeV}$ proton intensity from the ERNE instrument on SOHO (also at $L_{1}$ ) shown in Figure 11 confirms that this was the case. ERNE did not observe the initial rapid increase, only a slow increase similar to that observed by STEREO when inside the magnetic cloud. The two brief intensity peaks seen first at SOHO then at STEREO between $\sim 00-01$ UT on 15 December suggest that the profile included spatial features convected with the magnetic cloud.

Farrugia et al. (1993) noted a similar situation in which an SEP event onset was observed at IMP 8 (in Earth orbit) and at the ISEE-3 spacecraft (at $L_{1}$ ) as the leading edge of a magnetic cloud approached the Earth (Figure 12). The slower proton intensity increase observed inside the magnetic cloud was ascribed to the longer path length for particles arriving along helical field lines inside the magnetic cloud. A similar scenario might apply in the 14 December 2006 SEP event. In addition, we suggested above that particles arrived via the eastern leg of the magnetic cloud in this event, and this might also have influenced (possibly increased) the particle path length to the spacecraft in the magnetic cloud.

Without observations of the SEP event from a spacecraft outside the magnetic cloud (for example $\gtrsim 0.2 \mathrm{AU}$ downstream of the Earth), it is difficult to assess the impact of the 
magnetic cloud on the peak particle intensity near the Earth. The $60-100 \mathrm{MeV}$ proton intensity at STEREO certainly fell by around an order of magnitude on entry to the magnetic cloud (Figure 11), but it is unclear whether intensities later in the event were depressed by a similar factor compared to outside the magnetic cloud, and how this factor might have varied with energy. In particular, the lack of a particle intensity increase at the trailing edge of the magnetic cloud suggests that at this location, intensities inside and outside the cloud were similar. It is possible that the presence of the magnetic cloud may help to account for the smaller intensity of the 14 December particle event compared to that on 13 December despite the similar X-ray flare size and better (nominal) magnetic connection. On the other hand, the related CME observed by the LASCO coronagraphs on SOHO was considerably faster on 13 December (1774 $\left.\mathrm{km} \mathrm{s}^{-1}\right)$ compared to 14 December $\left(1042 \mathrm{~km} \mathrm{~s}^{-1}\right)$. This may have also contributed to the different event sizes since a faster CME-driven shock is expected to be a more efficient particle accelerator.

\section{Summary and Discussion}

We have discussed various interesting features of the energetic particle observations made by the STEREO A and B HETs and instruments on other near-Earth spacecraft during the SEP event of 14 December 2006.

Arrival of the leading edge of the magnetic cloud driving the shock associated with a solar event on 13 December abruptly terminated the rapidly increasing high energy proton event observed by the STEREO spacecraft. This rapid increase was not observed by spacecraft at $L_{1}$ that were already inside the magnetic cloud. A slower intensity increase occurred inside the magnetic cloud, possibly because proton arrival was delayed by the larger path length along helical magnetic fields (see Farrugia et al., 1993 for discussion of a similar situation). Another possibility is that field lines inside the magnetic cloud may have been poorly connected to the solar particle source region even though the magnetic cloud originated in, and hence might have been expected to be rooted in, the same solar active region. The fact that the particles appear to have traveled to the Earth via the eastern "leg" of the magnetic cloud may also have influenced the path length and connectivity. Thus, although we might expect the smooth fields and large particle mean free paths in magnetic clouds (e.g., Tranquille et al., 1987) to provide easy access for energetic particles to $1 \mathrm{AU}$, especially when the magnetic cloud and the particle event originate in the same active region, this is apparently not always the case. Without observations of the SEP event from a near-Earth spacecraft outside the magnetic cloud, it is difficult to assess the impact of the magnetic cloud on the peak particle intensity.

As other studies have noted (e.g., Mulligan et al., 2008 and Mulligan, Blake, and Mewaldt, 2008; Liu et al., 2008) unusual non-dispersive (i.e., spatial) particle intensity fluctuations extending in energy from tens of $\mathrm{keV}$ to $>100 \mathrm{MeV}$ were observed within the magnetic cloud. Our focus here has been on the remarkable differences in the intensity variations of 13.6-100 MeV protons observed by the HETs on the two STEREO spacecraft, despite the spacecraft being closely separated. We interpret these differences as evidence for particle anisotropies that were detected because the HETs were pointing in orthogonal directions due to STEREO-B being inverted at this stage of the mission. The observations suggest that energetic protons were $\sim$ isotropic in the high intensity regions of these variations (both instruments saw similar intensities despite their orthogonal viewing directions). In the lower intensity regions, particles were more anisotropic and arrived along the magnetic field from a direction close to the field of view of HET B, whereas HET A was observing particles with 
Figure 13 Three-dimensional figure of two helical flux tubes within a magnetic cloud, from Osherovich, Fainberg, and Stone (1999).
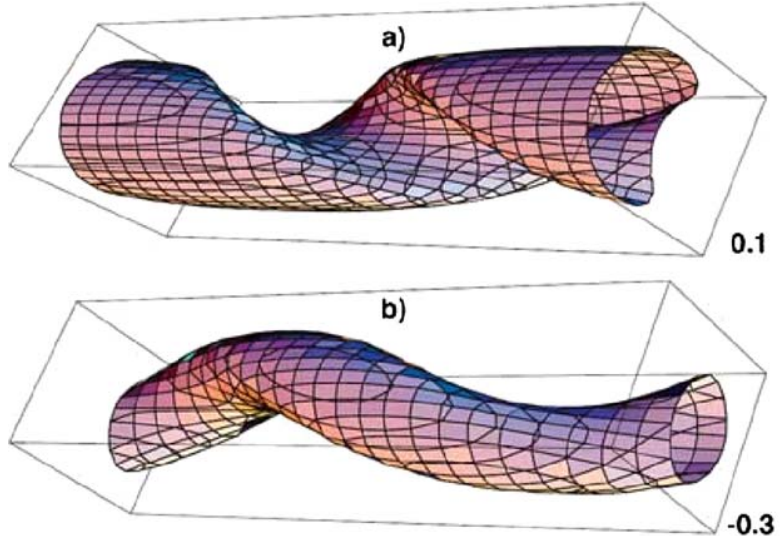

$\mathbf{z}$

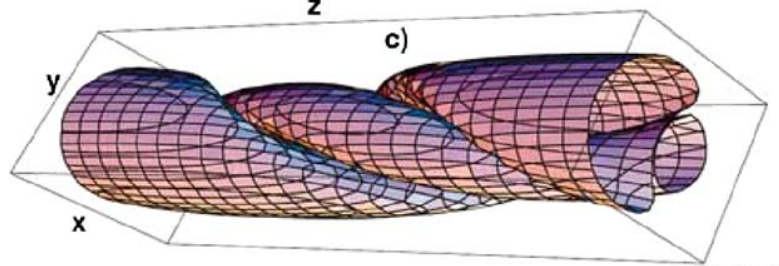

$(0.1,-0.3)$

pitch angles $\sim 90^{\circ}$, and hence measured a lower particle intensity. Anisotropy observations from Wind/EPACT show a similar picture albeit at lower energies $\sim 3 \mathrm{MeV}$. Suprathermal electron distributions observed by ACE/SWEPAM also appear to follow a similar pattern of narrower distributions in the regions of more anisotropic energetic ions.

The intensity/anisotropy variations were not associated with local magnetic field structures such as changes in field direction that might modulate the intensity observed by telescopes with fixed look directions. One possibility is that these variations were determined by propagation conditions along bundles of field lines between the Sun and the spacecraft that were not reflected in local solar wind conditions. In addition, studies by Fainberg et al. (1996) and Osherovich, Fainberg, and Stone (1999) have demonstrated that magnetic clouds may include multiple flux tubes that may be identified, for example, by abrupt changes in the field direction or in the slope of the anti-correlation between solar wind electron density and temperature within the magnetic cloud. We do not currently have solar wind electron parameters for the 14-15 December 2006 magnetic cloud and hence cannot assess in this way whether this magnetic cloud was divided into multiple flux tubes, and if so whether these were associated with changes in the energetic particle properties. There was an abrupt change in the magnetic field direction (current sheet) at $\sim 05$ UT on 15 December (Figure 5). Although it has been proposed that this feature might have been related to the dust trail of comet McNaught (Russell, Luhmann, and Szabo, 2007), we suggest that it was an internal feature of the magnetic cloud since, for example, the character of the rotation in $\theta_{\mathrm{B}}$ changed at this time, and the SWICS ion composition anomalies in the bottom panel of Figure 5 tend to be larger before this feature. However, this one feature clearly did not organize the more complicated variations in particle properties within the magnetic cloud (cf., Figure 4). Figure 13 (Osherovich, Fainberg, and Stone, 1999) illustrates how two interwoven magnetic flux tubes might have formed a magnetic cloud observed by the Ulysses spacecraft in June 1993. It is possible that a similar, possibly more complex, bundle of flux 
tubes formed the December 2006 magnetic cloud although the reconstruction of the magnetic cloud cross section by Liu et al. (2008) using the Grad - Shafranov technique suggests only a simple single flux rope structure, at least up to the current sheet in the middle of the magnetic cloud. Nevertheless, the energetic particle observations do suggest the existence of distinct flux tubes with diameters of $\sim 0.04 \mathrm{AU}$ within the magnetic cloud qualitatively similar to the picture in Figure 13. The small scale variations in particle properties on the order of 5-10 minutes suggest that these flux tubes may have had scale sizes ranging down to $\sim 0.003 \mathrm{AU}$. Alternatively, these small-scale variations might have been brief encounters with the boundaries of larger flux tubes.

The intensity modulations are reminiscent of the modulations seen in some impulsive solar particle events (e.g., Mazur et al., 2000; Chollet et al., 2007; Chollet and Giacalone, 2008) that have been attributed to crossing magnetic flux tubes that are or are not connected to a localized flaring region at the Sun. As in the present event, these modulations are occasionally observed inside ICMEs. Furthermore, the average 3.2 hour/0.03 AU time/distance scales for the intensity variations discussed by Mazur et al. (2000) are similar to those of the major variations in the 14 December event. However, as also noted by Mazur et al. (2000), it is difficult to see how flux tube connection to a particle source would account for intensity variations in a large gradual SEP event which presumably involved acceleration at an extended CME-driven shock. Given the large size of the particle source, it seems unlikely that adjacent groups of flux tubes in the magnetic cloud connected with, or did not connect with, the shock close to the Sun.

Mulligan et al. (2008) have also noted the similarity of the intensity variations in the 14 December event to the observations of Mazur et al. (2000) but suggest additional explanations for their origin. One proposal is that two populations of particles, one from the SEP event, the other accelerated or reflected by the shock that passed by on 14 December, might have been involved. Another is that particles may have been scattered sunward from structures in the sheath upstream of the magnetic cloud. However, given the wide range of energies over which the non-dispersive intensity fluctuations were observed (for example, protons from tens of $\mathrm{keV}$ to at least $100 \mathrm{MeV}$ ) and the observation of these fluctuations deep inside the magnetic cloud on field lines that are unlikely to have been connected to the shock (or even the sheath), we do not find these suggestions compelling. Furthermore, the bi-directional flows (in electrons) which Mulligan et al. (2008) mention in support of a scenario involving particles from both the shock and SEP event are not evident in the Wind proton data shown in Figure 9. In summary, the 14 December 2006 event demonstrates that the interplanetary transport of SEPs inside a magnetic cloud even with a near featureless magnetic field structure can be complicated by different propagation conditions/particle anisotropies and intensities along adjacent flux tubes. How this occurs is unclear.

Acknowledgements We thank Janet Luhmann of the University of California, Berkeley, for her steadfast support as the Principal Investigator of the STEREO/IMPACT investigation. The work at GSFC, Caltech and JPL was supported by NASA (Caltech and JPL were funded under subcontract SA2715-26309 from the University of California, Berkeley, under NASA Contract NAS5-03131). The ACE observations used in this paper are from the ACE Science Center (http://www.srl.caltech.edu/ACE/ASC/), and the STEREO magnetic fields were obtained from the STEREO archive at UCLA (http://www-ssc.igpp.ucla.edu/forms/stereo/). The SOHO/ERNE data were obtained from the Space Research Laboratory, University of Turku (http://www.srl. utu.fi/erne_data/).

Open Access This article is distributed under the terms of the Creative Commons Attribution Noncommercial License which permits any noncommercial use, distribution, and reproduction in any medium, provided the original author(s) and source are credited. 


\section{References}

Cane, H.V., Reames, D.V., von Rosenvinge, T.T.: 1988, J. Geophys. Res. 93, 9555.

Chollet, E.E., Giacalone, J.: 2008, Astrophys. J. 688, 1368.

Chollet, E.E., Giacalone, J., Mazur, J.E., Al Dayeh, M.: 2007, Astrophys. J. 669, 615.

Fainberg, J., Osherovich, V.A., Stone, R.G., McDowall, R.J.: 1996, In: Winterhalter, D., Gosling, J. T., Habbal, S. R., Kurth, W. S., Neugebauer, M. (eds) Solar Wind Eight, AIP Conf. Proc. 382, 554.

Farrugia, C.J., Richardson, I.G., Burlaga, L.F., Lepping, R.P., Osherovich, V.A.: 1993, J. Geophys. Res. 98, 15497.

Forman, M.: 1970, J. Geophys. Res. 75, 3147.

Klein, L.W., Burlaga, L.F.: 1982, J. Geophys. Res. 87, 613.

Liu, Y., Luhmann, J.G., Müller-Mellin, R., Schroeder, P.C., Wang, L., Lin, R.P., Bale, S.D., Li, Y., Acuña, M.H., Sauvaud, J.A.: 2008, Astrophys. J. 689, 563.

Marsden, R.G., Sanderson, T.R., Tranquille, C., Wenzel, K.-P., Smith, E.J.: 1987, J. Geophys. Res. $92,11009$.

Mazur, J.E., Mason, G.M., Dwyer, J.R., Giacalone, J., Jokipii, J.R., Stone, E.C.: 2000, Astrophys. J. 532, L79.

Mulligan, T., Blake, J.B., Mewaldt, R.A.: 2008, In: Proc. 30th Int. Cosmic Ray Conf. 1, 179.

Mulligan, T., Blake, J.B., Mewaldt, R.A., Leske, R.A.: 2008, Am. Inst. Phys. Conf. Ser. 1039, 162. doi:10.1063/1.2982440.

Osherovich, V.A., Fainberg, J., Stone, R.G.: 1999, Geophys. Res. Lett. 26, 401.

Palmer, I.D., Allum, F.R., Singer, S.: 1978, J. Geophys. Res. 83, 75.

Richardson, I.G., Cane, H.V.: 1995, J. Geophys. Res. 100, 23397.

Richardson, I.G., Cane, H.V.: 1996, J. Geophys. Res. 101, 27521.

Richardson, I.G., Reames, D.V.: 1993, Astrophys. J. Suppl. Ser. 85, 411.

Russell, C.T., Luhmann, J.G., Szabó, A.: 2007, In: American Geophysical Union, Fall Meeting 2007, abstract \#SM34A-08.

Tranquille, C., Sanderson, T.R., Marsden, R.G., Reinhard, R., Wenzel, K.-P.: 1987, J. Geophys. Res. 92, 6. von Rosenvinge, T.T., Reames, D.V., Baker, R., Hawk, J., Nolan, J.T., Ryan, L., Shuman, S., et al.: 2008, Space Sci. Rev. 136, 391. doi:10.1007/s11214-007-9300-5.

Zurbuchen, T.H., Richardson, I.G.: 2006, Space Sci. Rev. 123, 31. doi:10.1007/s11214-006-9010-4. 